\title{
Greater Curvature of the Stomach
}

National Cancer Institute

\section{Source}

National Cancer Institute. Greater Curvature of the Stomach. NCI Thesaurus. Code

C12262.

The lateral and inferior border of the stomach. Attached to it is the greater omentum. 